$11-2009$

\title{
Mechanics-based Analysis of Selected Features of the Exoskeletal Microstructure of Popillia Japonica
}

\author{
Liang Cheng \\ University of Delaware \\ Liyun Wang \\ University of Delaware
}

Anette M. Karlsson

Cleveland State University, a.karlsson@csuohio.edu

Follow this and additional works at: https://engagedscholarship.csuohio.edu/enme_facpub

Part of the Mechanical Engineering Commons

How does access to this work benefit you? Let us know!

\section{Original Citation}

Cheng, Liang., and Liyun Wang, et al. (2009). Mechanics-based Analysis of Selected Features of the Exoskeletal Microstructure of Popillia Japonica. Journal of Materials Research, 24(11), 3253-3267.

This Article is brought to you for free and open access by the Mechanical Engineering Department at EngagedScholarship@CSU. It has been accepted for inclusion in Mechanical Engineering Faculty Publications by an authorized administrator of EngagedScholarship@CSU. For more information, please contact library.es@csuohio.edu. 


\title{
Mechanics-based analysis of selected features of the exoskeletal microstructure of Popillia japonica
}

\author{
Liang Cheng, Liyun Wang, and Anette M. Karlsson ${ }^{\text {a) }}$ \\ Department of Mechanical Engineering, University of Delaware, Newark, Delaware 19716
}

\begin{abstract}
We explore key mechanical responses of the layered microstructure found in selected parts of the exoskeletons (pronotum, leg and elytron) of Popillia japonica (Japanese beetle). Image analyses of exoskeleton cross-sections reveal four distinct layered regions. The load-bearing inner three regions (exocuticle, mesocuticle, and endocuticle) consist of multiple chitin-protein layers, in which chitin fibers align in parallel. The exocuticle and mesocuticle have a helicoidal structure, where the stacking sequence is characterized by a gradual rotation of the fiber orientation. The endocuticle has a pseudo-orthogonal structure, where two orthogonal layers are joined by a thin helicoidal region. The mechanics-based analyses suggest that, compared with the conventional cross-ply structure, the pseudo-orthogonal configuration reduces the maximum tensile stress over the exoskeleton cross-section and increases the interfacial fracture resistance. The coexistence of the pseudo-orthogonal and helicoidal structures reveals a competition between the in-plane isotropy and the interfacial strength in nature's design of the biocomposite.
\end{abstract}

\section{INTRODUCTION}

The hard exoskeleton of a beetle is an important part of the arthropod's body. It has outstanding structural properties with multifunctional capabilities, such as supporting the body weight, filtering chemicals, and resisting external loads (e.g., enemy attack). The multifunctional properties are obtained by constructing an intricate, hierarchical structure, using primarily the biopolymers chitin along with associated proteins. ${ }^{1,2}$ Thus, the exoskeleton is a multifunctional biological composite. Unlike the mineralized exoskeletons of crustaceans such as Homarus americanus (American lobster) and Callinectes sapidus (Atlantic blue crab), ${ }^{3}$ the majority of insects do not have a significant amount of minerals (e.g., calcite) in their exoskeletons. Minerals would make insects too heavy to fly. ${ }^{1,2}$

The exoskeleton of a beetle is typically divided into four regions: the epicuticle, exocuticle, mesocuticle, and endocuticle. ${ }^{1.4}$ The outermost region, the epicuticle, is the thinnest region (typically less than $1 \mu \mathrm{m}$ ) and consists of lipids and proteins. It acts as a diffusion barrier and is not load-bearing. ${ }^{1.2}$ The remaining exoskeleton (exocuticle, mesocuticle, and endocuticle) provides the load-bearing capacity. Here, the major building materials are chitin fibers, and the associated proteins are supplemented with lipids, pigments, inorganic materials, and water. ${ }^{1,2,5}$ Chitin has an elastic modulus up to $100 \mathrm{GPa}^{6}{ }^{6,7}$ and even higher

\footnotetext{
address all correspondence to this author.

e-mail: karlsson@udel.edu

DOI: 10.1557/JMR,2009.0409
}

values have been reported (the order of $100-200 \mathrm{GPa}^{8.9}$ ). and forms microfibrils with a diameter of approximately 2 to $3 \mathrm{~nm}^{10}$ The microfibrils and the surrounding protein matrix (elastic modulus less than $1 \mathrm{GPa}^{6}$ ) form a composite structure. ${ }^{1,2,11}$ Furthermore, bundles of microfibrils are assembled to form the so-called "macrofibrils," which can be observed under electron microscopy. ${ }^{2,12}$ The macrofibrils usually align with each other to form layers parallel to the exoskeleton surface, and these layers are organized with specific patterns to construct the exoskeletons. It is interesting to note that similar patterns can be found in species ranging from insects, such as beetles, to crustaceans, such as crabs, lobsters, crayfish, and to (for example) ancient species (but still with existing), such as "armored fish." "-3,13-18

Because the exoskeleton consists of relatively simple constituent materials (chitin, proteins, water and/or minerals), the complex hierarchical structure is crucial to achieve the excellent mechanical properties and versatility of the exoskeletons. ${ }^{5.8 .13,19}$ The relationship between the microstructure and the mechanical responses have been studied both experimentally and theoretically in a variety of exoskeletons including gastropod molluscs, ${ }^{19.20}$ armored fish, ${ }^{13}$ and arthropods. ${ }^{3,5.14-17,21-23}$ A common configuration of arthropod exoskeletons, including crustaceans, ${ }^{3,16,23}$ and insects, ${ }^{5,14,15,17,22}$ is the chitin-based layered structure forming a "helicoidal" pattern. ${ }^{2,4,12,16}$ In a previous study, ${ }^{3}$ we showed that cuticles with helicoidal structures exhibit nearly isotropic mechanical response when subjected to external in-plane 
loads. ${ }^{3}$ In addition to the helicoidal structure, there are additional structural arrangements, such as the cross-ply pattern $^{1,2,17}$ and a pattern in which fibers are orientated perpendicular to the surface, ${ }^{22}$ that contribute and influence the overall mechanical response. The transverse pore canal fibers that we found in lobsters and crabs have been shown to increase the interlaminar strength of the layered exoskeletons. ${ }^{3}$

The objective of this study is to investigate the unique hierarchical microstructure and its implications on the mechanical behavior of the exoskeleton of Popillia japonica. Although this is now a common insect in the United States, to the knowledge of the authors, its microstructure has not been investigated previously. We ultimately strive to understand nature's structural design principles and to use these designs in creating and fabricating bioinspired man-made materials and structures. In the following sections, we first examine the microstructure of the $P$. japonica exoskeletons at three locations of the arthropod body (pronotum, leg, and elytron) by using scanning and transmission electron microscopes (SEM and TEM). The detailed microstructure of the $P$. japonica is established by analyzing the images, augmenting the current knowledge on the exoskeletal microstructure of insect exoskeleton (see for example Refs. 4, 14, 17, and 22). Based on the image analyses, mechanics-based modeling, using the finite element analysis and analytical methods, is then performed. The mechanics-based simulations and parametric studies reveal the importance of the morphology on the mechanical behavior of the exoskeleton. With our ultimate goal being to develop a man-made, bioinspired structure, the mechanics-based evaluation is presented in general, nondimensionalized terms and does not focus on capturing the exact mechanical properties of the $P$. japonica.

\section{IMAGE ANALYSIS}

\section{A. Sample preparations}

The exoskeletons investigated are from species of the $P$. japonica (Japanese beetle) that were collected during July and August 2008 in local gardens and parks in Newark, Delaware, and stored in $70 \%$ ethanol alcohol (ETOH) at $4{ }^{\circ} \mathrm{C}$. Representative samples of exoskeletons were obtained from three locations of the $P$. japonica body: pronotum (back cover) and leg and elytron (wing cover) (Fig. 1). The samples were divided into two groups (10 samples per group) for each location and prepared for either SEM or TEM:

(i) SEM: cuticles from the first group were placed in 95\% ETOH immediately after being dissected from the investigated locations. The cuticles were then dehydrated in $100 \%$ ETOH and $100 \%$ anhydrous ETOH for $30 \mathrm{~min}$ each, followed by critical point dehydration (Autosamdri 815B, Seris A; Tousimis Research Corpo-

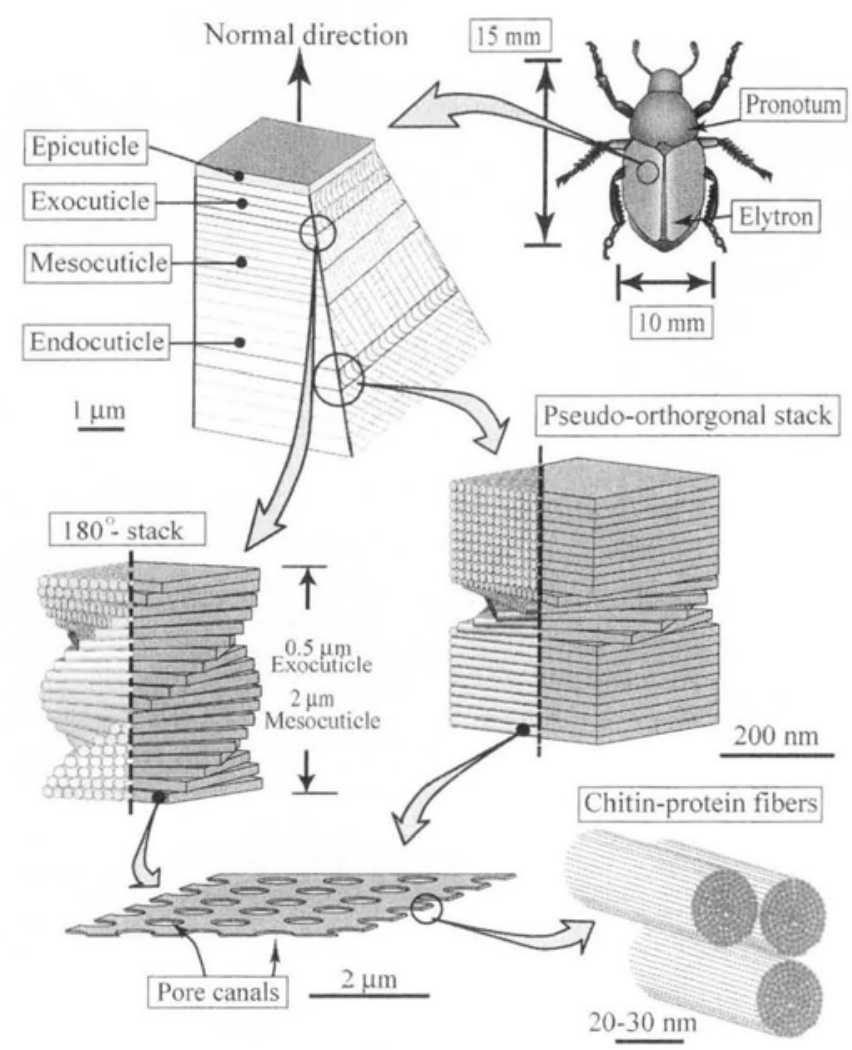

FIG. 1. Schematics of the hierarchical structure of the exoskeleton of a $P$. japonica. The outer layer (epicuticle) acts as a diffusion barrier. The exocuticle, mesocuticle, and endocuticle are the main loadbearing structures composed of fibrous chitin-protein fibers organized as either helicoidal (exo- and mesocuticle) or pseudo-orthogonal (endocuticle) structures.

ration, Rockville, MD), ${ }^{3}$ before the samples were fractured by using forceps and single-edge razor blades. Due to the difficulty in fracturing the small samples, oblique sections were commonly obtained. The fractured samples were mounted onto aluminum stubs with conductive silver paint (Electron Microscopy Sciences, Hatfield, PA), coated with Gold/Palladium (Denton Bench top Turbo III sputter-coater; Denton Vacuum LLC, Moorestown, NJ), and observed by using a SEM (Hitachi 4700 FESEM; Hitachi Ltd., Tokyo, Japan).

(ii) TEM: following the method of Lindley, ${ }^{24}$ cuticles from the second group were placed in $80 \%$ ETOH immediately after dissection. The cuticles were then left in $80 \%$ ETOH with $1 \% \gamma$-glycidoxypropyltrimethoxysilane overnight as an adhesion promoter between the specimen outer surface and the embedding resin. The treated samples were placed in $100 \%$ LR White resin at $4{ }^{\circ} \mathrm{C}$ for three consecutive days and polymerized at $60{ }^{\circ} \mathrm{C}$ for $48 \mathrm{~h}$ in an oxygen-free environment in a standard incubator (Barnstead Thermolyne, Type 19200; H\&C Thermal Systems, Columbia, MD). Blocks of resin containing the samples were trimmed and microwaved for $2 \mathrm{~min}$ in $60 \mathrm{~mL}$ of water to ensure cross-polymerization of the resin within the samples. ${ }^{24}$ The samples were then sectioned by using a 
microtome (Reichert-Jung Ultracut E ultramicrotome; Labequip Ltd., Markham, ON, Canada) to obtain sections (60-80-nm thick) in three directions: cross-sections, sections parallel to the outer surface, and oblique sections. These sections were collected onto formvar carbon-coated copper grids, stained with methanolic uranyl acetate and lead citrate, ${ }^{25}$ and observed with a TEM at an accelerating voltage of $80 \mathrm{kV}$ (Zeiss CEM 902 TEM; Carl Zeiss AG, Oberkochen, Germany).

\section{B. Microstructural observations}

Observations of the $P$. japonica exoskeletons reveal an overall similar morphology for all the investigated locations, including the four distinct layered regions: the epicuticle, exocuticle, mesocuticle, and endocuticle (Figs. 1 and 2). A transverse pore canal system is also observed, running perpendicular through the exoskeletons (Fig. 3). The key features and measurements of the exoskeletons are summarized in Table I and discussed in detail below.

The overall thickness of the exoskeleton of the $P$. japonica varies from 18 to $32 \mu \mathrm{m}$ at the three locations, with the thickest to the thinnest being pronotum (28-32 $\mu \mathrm{m})$, leg $(19-23 \mu \mathrm{m})$, and elytron $(18-20 \mu \mathrm{m})$. The thicknesses of the epicuticle, exocuticle, mesocuticle, and endocuticle increase monotonically from the outer surface and inward (Table I). The epicuticle is not load-bearing and will not be discussed further. We focus on the loading-bearing regions (the exocuticle, mesocuticle, and endocuticle) in this study. The morphology of the endocuticle is fundamentally different from those of the exo- and mesocuticle (Fig. 2). We first present the helicoidal structural patterns found in the exo- and mesocuticles and then describe the unique pseudo-orthogonal pattern found in the endocuticle. (Analyzing the structures using SEM and TEM does not reveal how the exoskeleton develops two characteristic morphologies. Examination at selected times during the development of the exoskeleton could help establish the mechanisms for the formation of the two morphologies. However, this theory is beyond the scope of the current work.) We also briefly discuss the pore canal system.

\section{Helicoidal structure}

The exocuticle and mesocuticle are both constructed by a helicoidal structure, which is sometimes referred to as the "Bouligand" structure. ${ }^{2,12}$ The exocuticle is thinner than the mesocuticle (see Fig. 2 and Table I). For example, in the elytron the exocuticle thickness is 1.5 to $2 \mu \mathrm{m}$ and the mesocuticle thickness is 7 to $7.5 \mu \mathrm{m}$. Both regions consist of multiple chitin-protein layers parallel to the surface, with chitin-protein macrofibrils arranged

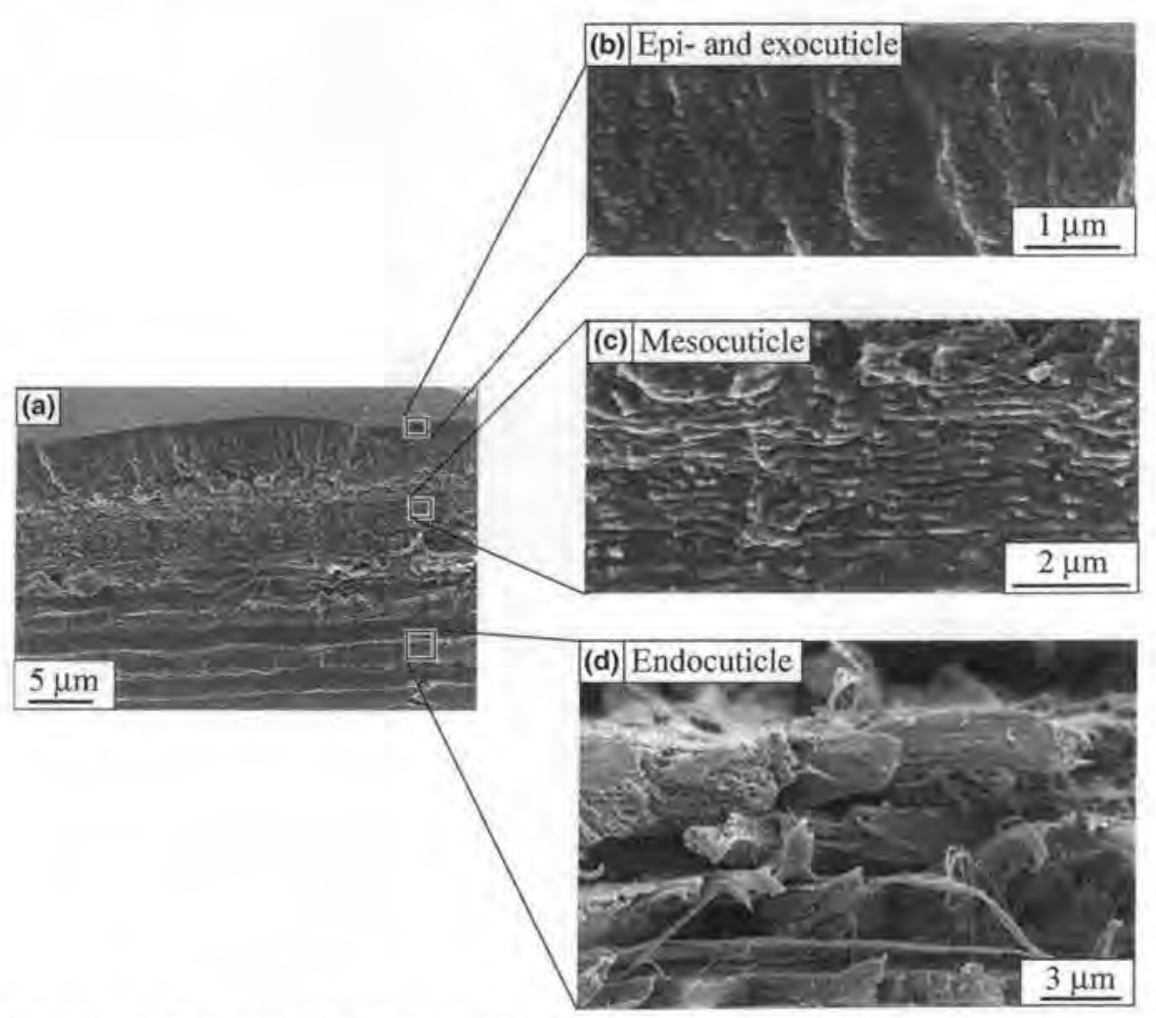

FIG. 2. SEM images of the exoskeleton (elytron) of a $P$. japonica. (a) Overview of the cross-section; (b) epicuticle and exocuticle ( $\sim 2-\mu \mathrm{m}$ thick); (c) mesocuticle (7-7.5- $\mu \mathrm{m}$ thick); and (d) endocuticle (10-11- $\mu \mathrm{m}$ thick). The stacking of two orthogonal layers is shown in the endocuticle. 

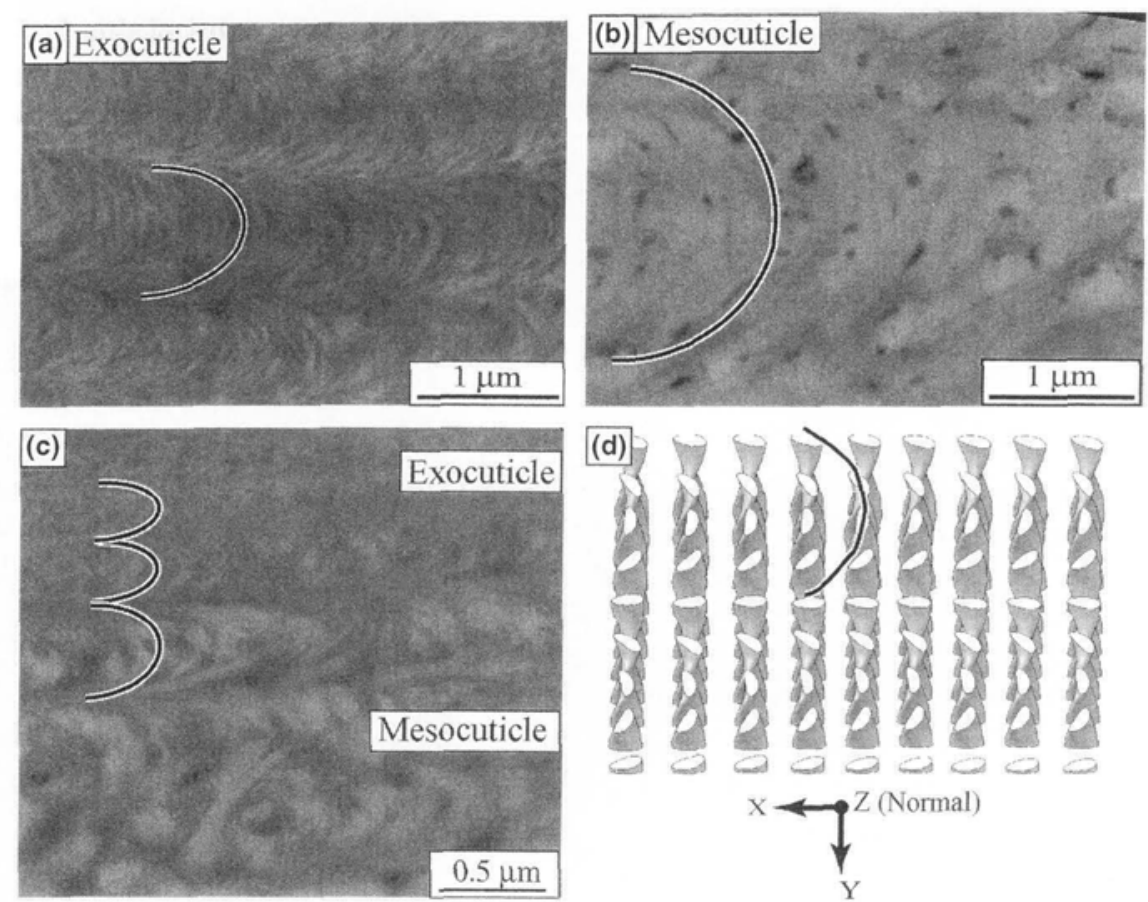

FIG. 3. The helicoidal structure (Bouligand-structure) as observed in TEM images of the P. japonica elytron. (a) The parabolic pattern of the chitin-protein fibers from the oblique sections of the exocuticle; (b) the parabolic pattern of the pore canals from the oblique sections of the mesocuticle; (c) oblique section of the exo- and mesocuticle, characterized by parabolic patterns, but with larger spans of parabolic profiles in the mesocuticle compared to those in the exocuticle (the size of each parabola is larger than the true thickness of corresponding $180^{\circ}$ stack since it is observed from an oblique section); (d) a schematic representation of the twisted ribbon morphology ${ }^{3}$ of the pore canal and the parabolic pattern of pore canals as observed from the oblique section [seen in (b)].

TABLE I. Summary and comparison of exoskeleton structure of samples from three positions of the $P$. japonica: pronotum, elytron, and leg. Note: pore canals extend throughout the structure, excluding the epicuticle.

\begin{tabular}{lllll}
\hline \hline & \multicolumn{2}{c}{ Pronotum } & Leg & Elytron \\
\hline Epicuticle & Thickness $[\mu \mathrm{m}]$ & $0.3-0.7$ & $0.1-0.2$ & $0.1-0.2$ \\
Exocuticle & Thickness $[\mu \mathrm{m}]$ & $6.5-7$ & $4-5$ & $1.5-2$ \\
& Structural pattern & Helicoidal $\sim 0.1 \mu \mathrm{m}$ per $180^{\circ}$ stack & Helicoidal $\sim 0.1 \mu \mathrm{m}$ per $180^{\circ}$ stack & Helicoidal $\sim 0.15 \mu \mathrm{m}$ per $180^{\circ}$ \\
& & & stack \\
Mesocuticle & Thickness $[\mu \mathrm{m}]$ & $7-8$ & 5 & $7-7.5$ \\
& Structural pattern & Helicoidal $\sim 0.15 \mu \mathrm{m}$ per $180^{\circ}$ & Helicoidal $\sim 0.15-0.2 \mu \mathrm{m}$ per $180^{\circ}$ & Helicoidal $\sim 0.25 \mu \mathrm{m}$ per $180^{\circ}$ \\
& stack & stack & $10-11$ \\
Endocuticle & Thickness $[\mu \mathrm{m}]$ & $14-16$ & $10-13$ & Pseudo-orthogonal $\sim 1 \mu \mathrm{m}$ per \\
& Structural pattern & Pseudo-orthogonal $\sim 2 \mu \mathrm{m}$ per & Pseudo-orthogonal $\sim 2 \mu \mathrm{m}$ per & unidirectional layer \\
& & unidirectional layer & unidirectional layer & layer \\
& & & &
\end{tabular}

parallel within each layer, similar to the multiple layered exoskeletons of $H$. americanus and $C$. sapidus that we investigated in our previous study. ${ }^{3}$ In this structure, the layers stack successively on each other, with each layer rotated unidirectionally by a small angle about its normal direction relative to the adjacent layer. The repeating unit is termed " $180^{\circ}$-stack" in Fig. 1. The stacking sequence results in an apparent parabolic pattern in the oblique sections of the cuticles. Series of parabolic curves can be observed under TEM from oblique sections [Fig. 3(a)], with each "full parabola" corresponding to an $180^{\circ}$-degree accumulated rotation of layers. ${ }^{2,3,12}$
Despite sharing the same structural patterns, the helicoidal $180^{\circ}$ stacks in the exocuticle are denser and thinner than those in the mesocuticle (Table I). For example, in the elytron the thickness of an $180^{\circ}$ stack in the exocuticle is approximately $0.15 \mu \mathrm{m}$, compared to $0.25 \mu \mathrm{m}$ in the mesocuticle [see Figs. 2 and 3(c) and Table I].

\section{Pseudo-orthogonal structure}

The endocuticle is the thickest region, constituting more than half of the total thickness of the exoskeletons for the three studied locations [Fig. 2(a)]. It is interesting 
to note that the endocuticle has a distinctly different structure from the helicoidal structure in the exo- and mesocuticles. Here, the chitin-protein layers stack together unidirectionally, forming a relatively thick layer [e.g., approximately $1-\mu \mathrm{m}$ thick in the elytron (Table I)]. Successive unidirectional layers stack onto each other orthogonally. Consequently, it resembles the common man-made cross-ply laminate [Fig. 2(d)]. However, there is a thin transitional region (e.g., 0.1-0.2- $\mu \mathrm{m}$ thick in the elytron) between two adjacent orthogonal layers, where the fibers in the transitional region appeared to be arranged helicoidally. The transitional region bridges the orthogonal structure, with a helicoidal $90^{\circ}$ rotation [Fig, 4(c)]. The overall structure was named the "pseudoorthogonal structure" by researchers who found a similar structure in adult locust and beetle cuticles. ${ }^{17.21} \mathrm{We}$ will adopt this nomenclature here.

The orthogonal stacking sequence is evident under SEM observation [Fig, 4(a)]. The microstructure of the thin transitional region and the corresponding parabolic pattern in oblique sections due to its helicoidal pattern can be observed via TEM imaging [Fig. 4(b)]. A schematic reconstruction of a cross-ply block with a helicoidal transition zone [Fig. 4(c)], subjected to an oblique cut [Fig. 4(d)], results in a pattern [Fig. 4(e)] corresponding to what is observed in the oblique TEM sections [Fig. 4(b)].

\section{Pore canals and pore canal fibers}

Through-the-thickness pore canal system is an important characteristic of the beetle cuticle, similar to the $C$. sapidus and $H$. americanus. ${ }^{3}$ The pore canals are oval-shaped, formed by the opening and closing of the chitin-protein macrofibril branches in each layer, resulting in a "twisted ribbon" geometry through the thickness of the two helicoidal regions. ${ }^{3,26}$ Similar to the $180^{\circ}$ stacks, a parabolic pattern of pore canal sections are

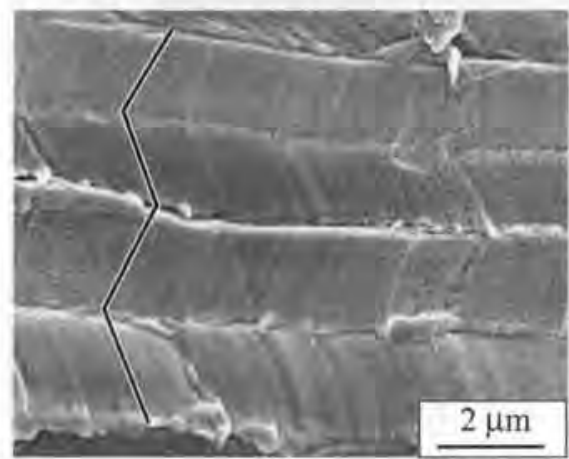

(a)

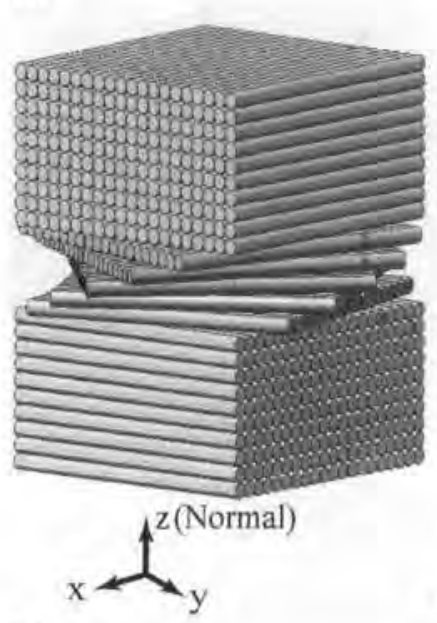

(c)

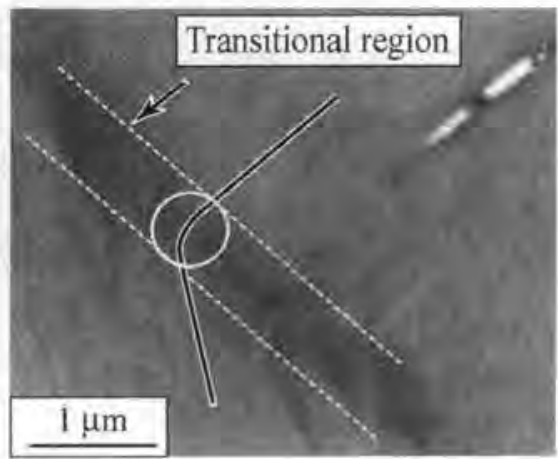

(b)

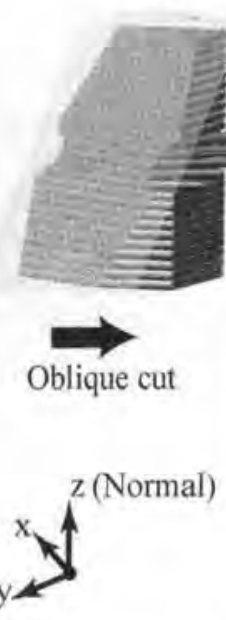

(d)

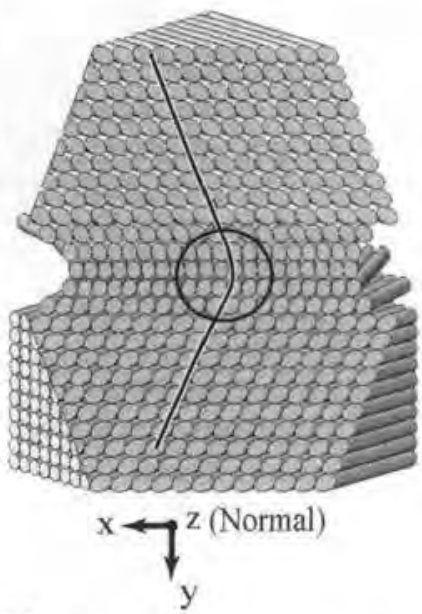

(e)

FIG. 4. The pseudo-orthogonal partern of the chitin-protein fibers in the endocuticle. (a) A zigzag pattern seen in a SEM image of an oblique section of the endocuticle from the $P$. japonica elytron; (b) a TEM image of an oblique section of the endocuticle, suggesting a helicoidal transitional region (parabolic pattern) between the two orthogonal layers (the size of the transitional region appears to be thicker than the true thickness since it is observed from an oblique section); (c) a schematic representation of the pseudo-orthogonal pattern: the two orthogonally stacked unidirectional layers each consists of parallel fibers. A thin transition region assembling a helicoidal structure (Bouligand-structure) joins the two orthogonal layers; (d) an oblique cut in the pseudo-orthogonal block; (e) the side view of the oblique cut displays the zigzag and parabolic pattern seen in (a) and (b). 
observed under TEM from the oblique sections of the cuticle. This feature is particularly evident in the mesocuticle region [Figs. 3(b) and 3(d)].

\section{THE STRUCTURES' IMPLICATIONS ON MECHANICAL BEHAVIOR}

As described above, $P$. japonica exoskeletons are natural biocomposites constructed by a protein matrix reinforced with chitin fibers. The most distinctive structural feature found in the exoskeletons is that the chitin-protein layers (lamina) are organized in either helicoidal or pseudo-orthogonal patterns, as described in the previous section. The mechanical response of the helicoidal structure and of the pore canal system was discussed in our previous study. ${ }^{3}$ In summary, a helicoidal structure containing a relatively large number of layers results in a high level of in-plane isotropy, providing isotropic structural responses in the plane of loading. Furthermore, the pore canal system increases the interlaminar strength of the layered structure constituting the exoskeleton. ${ }^{3}$

In the following text, we focus our modeling effort on the pseudo-orthogonal structure found in the endocuticle of the P. japonica exoskeleton. Models for the mechanical analysis are based on our understanding of the microstructure derived from our image analysis and from literature. The distinctive pseudo-orthogonal pattern is incorporated into the models to explore its implications on the mechanical behavior of the functional structure. It is well established that the material properties of biocomposites are influenced by many factors. Besides the complex hierarchical structures, the composition, and spatial distribution of the materials, $5,27,28$ interactions among different components (e.g., extent of chitin-protein interaction, strength of interphase bonding),,$^{5,29,30}$ and hydration status ${ }^{5,15,22,31}$ all directly influence the material properties. More elaborate multiscale modeling involving nonlinear mechanism and interfacial molecular interactions, ${ }^{32,33}$ as well as experimental characterization of the material composition and spatial distribution along with measuring the mechanical properties ${ }^{20,22,27,31}$ are needed to fully characterize the material structureproperty relationship of the investigated exoskeleton. However, to elucidate how the microstructure influences the mechanical response, we use the assumptions of linear elasticity and continuum mechanics. In this approach, all factors that affect the material properties are condensed into the "apparent" material properties and do not have to be modeled explicitly. The elastic material parameters are derived from available data based on classic continuum mechanics and laminate composite theory, which satisfactorily represent the structure's mechanical characteristics at lamina scale. ${ }^{3}$ Moreover, our results are presented in the nondimensionalized forms to emphasize the coherent structure-property relationship rather than the absolute values of individual parameters. This approach highlights the effect the microstructure has on the overall mechanical response and will provide important guidelines when developing man-made, bioinspired structures.

\section{A. Models and model parameters}

The major difference between the conventional crossply pattern and pseudo-orthogonal pattern is the thin helicoidal transitional region that joins two adjacent orthogonally stacked unidirectional layers. We construct two models to compare the two structures:

(i) A "conventional" cross-ply configuration, characterized by successive unidirectional fiber-reinforced laminae stacked together with $90^{\circ}$ directional change about the normal direction. For simplicity, only two layers $\left(0\right.$ and $90^{\circ}$ orientation) of equal thickness are included in the model [Fig. 5(a)].

(ii) A pseudo-orthogonal configuration, where a helicoidal transitional region is inserted between the two orthogonal ( 0 and $90^{\circ}$ orientation) layers [Fig. 5(b)]. Two sets of submodels are used:

(a) constant thickness of the transitional region (the same thickness as the orthogonal layer), where the number of transitional lamina are varied from 1 to 50 lamina, discussed below (the thickness of each individual lamina changes), and

(b) variable thicknesses of the transitional region, where the number of transitional lamina is constant (we selected 10 lamina, discussed below). The thickness of the transitional region corresponding to 10,20, and 33\% of the overall thickness is investigated.

Each submodel assumes a uniform lamina thickness and rotation angle between adjacent laminae in the helicoidal transitional region. The number of laminae in the transitional region cannot be directly identified from our TEM images. Based on the thickness of the transitional region (approximately $0.1-0.2 \mu \mathrm{m}$ in the elytron) and the thickness of a single lamina (approximately $20-40 \mathrm{~nm}$, assuming a lamina consists of one layer of macrofibrils), we estimate the number of lamina to approximately 10 . The number of transitional lamina corresponds to a specific angle of rotation between adjacent laminae. To predict the influence of the transitional region on the mechanical response of the structure, the number of the laminae is varied from 1 to 50 in the transitional region (model iia above). The thickness of the helicoidal region is enlarged from the original biolaminate configuration [Fig. 5(b)] to (i) highlight and focus on the mechanical response of the transitional region, which distinguishes the pseudo-orthogonal from the traditional cross-ply configuration, and (ii) possibly extend the interpretation to potential engineering applications with 


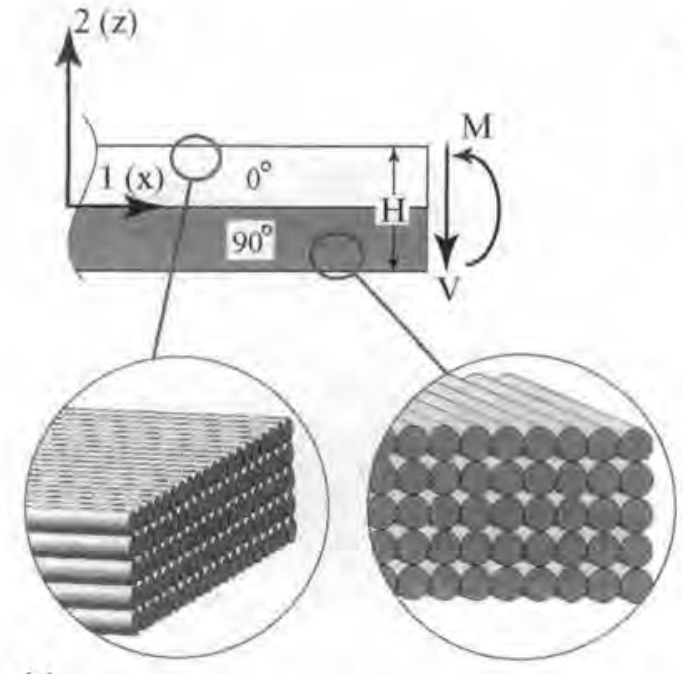

(a)

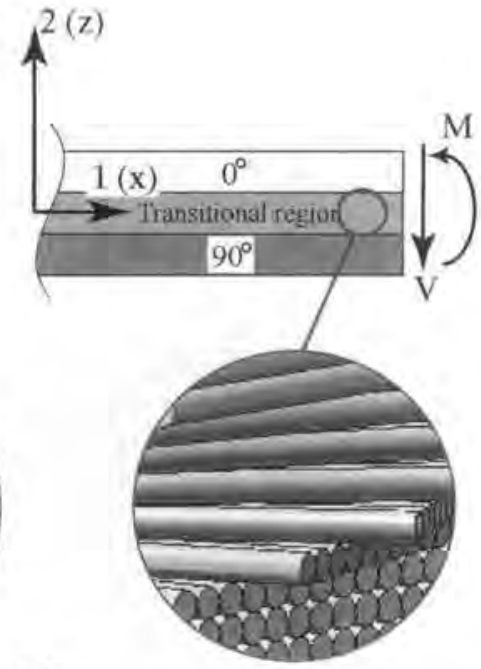

(b)

FIG. 5. Models of (a) the traditional cross-ply laminate and (b) bioinspired pseudo-orthogonal laminate of thickness, $H$. The cross-sections of the laminates are subjected to the general load of bending moment $M$ and shear force $V$ (load per unit width). The schiematic enlargement of each region indicates the fiber orientation in the corresponding region.

various transitional region ratios as demanded by functionality or manufacturing.

To elucidate and compare the mechanical response of these structures, we investigate the stress and strain fields of both models of a constant overall laminate thickness under a general load configuration, as shown in Fig. 5. In the linear elastic range, the equations relating the shear stresses (strains) to the shear force are uncoupled from the equations relating normal stresses (strains) to the bending moment. In other words, within the linear elastic range, shear stresses (strains) and normal stresses (strains) can be determined without the knowledge of how the shear force is related to the bending moment at the cross-section considered. Therefore, the study of the structure's mechanical response will not be confined to a specific external load; instead, we will simply consider a cross-section subjected to the arbitrary shear force $V$ and bending moment $M$ (Fig. 5).

As previously mentioned, we use a continuum mechanics approach, implicitly incorporating all relevant factors, such as interaction between components (e.g., the interaction between chitin fiber and protein matrix), the specific component proportion, and the water contents, into the apparent material properties. ${ }^{3}$ Thus, only the lamina properties are used in the following analyses. The lamina properties are characterized by a significantly higher elastic stiffness in the fiber direction compared to the two transverse directions (in-plane and out-of-plane) due to the high stiffness of the reinforcing fiber along the fiber direction. The normalized nominal lamina material properties are listed in Table II. The elastic properties of the stacking laminae (orientated from 0 to $90^{\circ}$ ) are obtained based on the classic laminate theory. (A brief summary of the "classic laminate
TABLE II. Normalized nominal lamina elastic properties in the global coordinate system.

\begin{tabular}{lrrrl}
\hline \hline Orientation & $E_{1}$ & $E_{2}$ & $G_{12}$ & $V_{12}$ \\
\hline $0^{\circ}$ & 25 & 1 & 0.5 & 0.25 \\
$90^{\circ}$ & 1 & 25 & 0.2 & 0.3 \\
\hline \hline
\end{tabular}

theory" used here is presented in Appendix A.) Although comprised of the same material components, the properties of the laminae measured in a global coordinate system (denoted as 1,2,3) depend on the local orientation of the fibers (Appendix A). Furthermore, due to the asymmetry resulting from the helicoidally transitional region, the bending-stretching coupling stiffnesses $B_{i j}$ are retained in the constitutive equations, and thus a closed form analytical solution is not available. ${ }^{34}$ Finite element analysis is implemented by using the commercial FE software ABAQUS ${ }^{35}$ to model the problem. For simplicity, plane strain conditions are assumed and the model is constructed with 1000 (length) $\times 300$ (thickness) plane-strain elements, CPE8R. ${ }^{35}$

Stress and strain (normal and shear) due to the crosssectional load per unit width of bending moment $M$ and shear stress $V$ are investigated (Fig. 5). The stresses are presented in nondimensionalized forms according to the following:

$$
\hat{\sigma}_{1}=\frac{H^{2}}{M} \sigma_{1} ; \hat{\tau}_{12}=\frac{H}{V} \tau_{12}
$$

where $H$ is the laminate thickness, $\sigma_{1}$ and $\tau_{12}$ are the normal stress and transverse shear stress respectively, and $\hat{\sigma}_{1}$ and $\hat{\tau}_{12}$ are nondimensionalized normal stress and shear stress. 
Interfacial strength is a major concern of laminated composites. Thus, in addition to establishing the stress and strain fields, the interfacial strength of the structure will be qualitatively addressed with a simple linear elastic fracture mechanics (LEFM) approach.

\section{B. Results and discussion}

\section{Stresses and strains in the laminate}

The numerical results reveal a clear advantage of the pseudo-orthogonal compared with the cross-ply configuration by distinct redistributions of the stress and strain fields within the structure, as summarized in Figs. 6 and 7.

First, consider the normal stress and strain distribution over the laminate cross-section. We show with a simple analytical approach in Appendix B that the normal strain must be continuous, but that the normal stress cannot be continuous over the interface between two laminates (within the assumption of continuum mechanics). This discovery is captured by the numerical simulations, where the normal strain distribution is continuous over the interfaces for both configurations [Fig. 6(b)] and the discontinuity of the normal stress appears as a "jump" in the curves [Fig. 6(a)]. The conventional cross-ply laminate exhibits a large jump; that is, there is a large difference between the stresses over the interface. [We note that within the continuum mechanics approach, the interface is infinitely small. If a multiscale approach were adopted, this interface would have a defined thickness. The discontinuity seen in the current simulations would typically be absorbed by the interface in terms of a steep gradient over its thickness. However, this reaction would only change the details, not the overall response of the structure investigated (assuming that the materials and structural properties are consistent between the approaches).] This effect is due to the large stiffness mismatch across the interface (Appendix B). For the pseudo-orthogonal laminate, the discontinuities remain, but the jumps are significantly smaller and decrease with the increasing number of transitional layers (e.g., decreasing rotation angle between the transitional helicoidal laminae) [Fig. 6(a)]. The decrease is directly related to the decrease in stiffness mismatch over the adjacent laminae. Thus, for the pseudo-orthogonal structure, the transitional region reduces the discrete change in the stiffness between the orthogonal layers and therefore "smoothes" the stress discontinuities. Increasing the number of laminae in the transitional region decreases the rotation angle. This effect, in turn, decreases the change in stiffness across the interface. Consequently, the normal stress distribution through the thickness approaches a continuous profile when the number of transitional laminae increases (keeping the overall thickness constant).
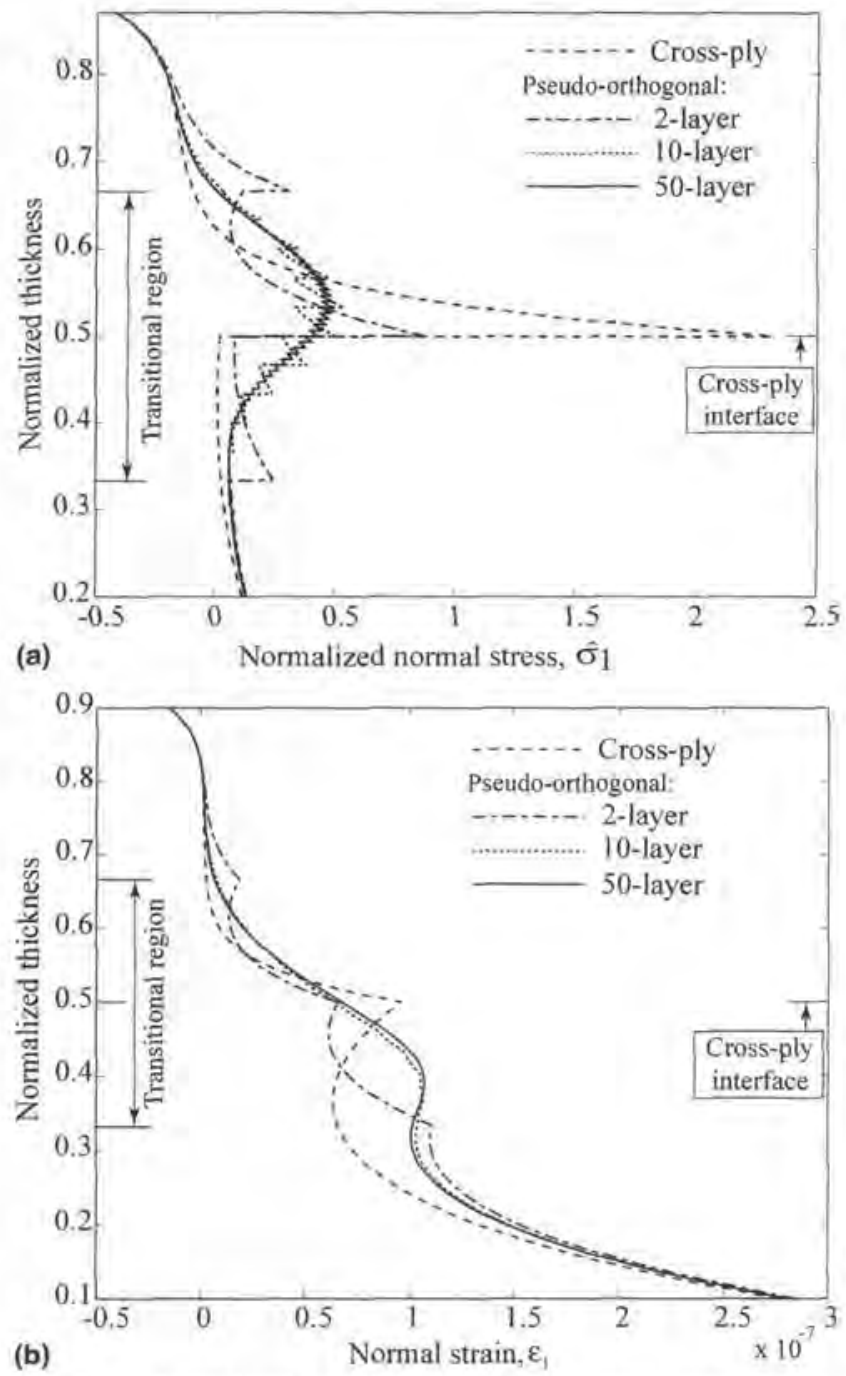

FG. 6. (a) Normalized normal stress and (b) normal strain distribution over the cross-section of the laminate with selected numbers of lamina in the transitional region.

The maximum tensile stress along the laminate cross-section is significantly reduced for the pseudoorthogonal structure [Figs. 6(a) and 8]. The redistribution of the normal stress field shifts the maximum load from its location in the $0^{\circ}$-orientation layer near the interface to the laminae in the transitional region. Therefore, the transitional region effectively reduces the maximum tensile stress, resulting in a more uniform tensile stress distribution. The results also suggest that the maximum tensile stress is reduced with the increasing number of transitional laminae, but no further reduction appears to be achieved beyond approximately 10 laminae (Fig. 8). This result is interesting, because our experimental observation suggests that the transition region $(\sim 0.2-\mu \mathrm{m}$ thick) consists of approximately 10 laminae of macrofibrils $(\sim 20 \mathrm{~nm})$. Consequently, our simulations are consistent with the observed structures created by nature. 

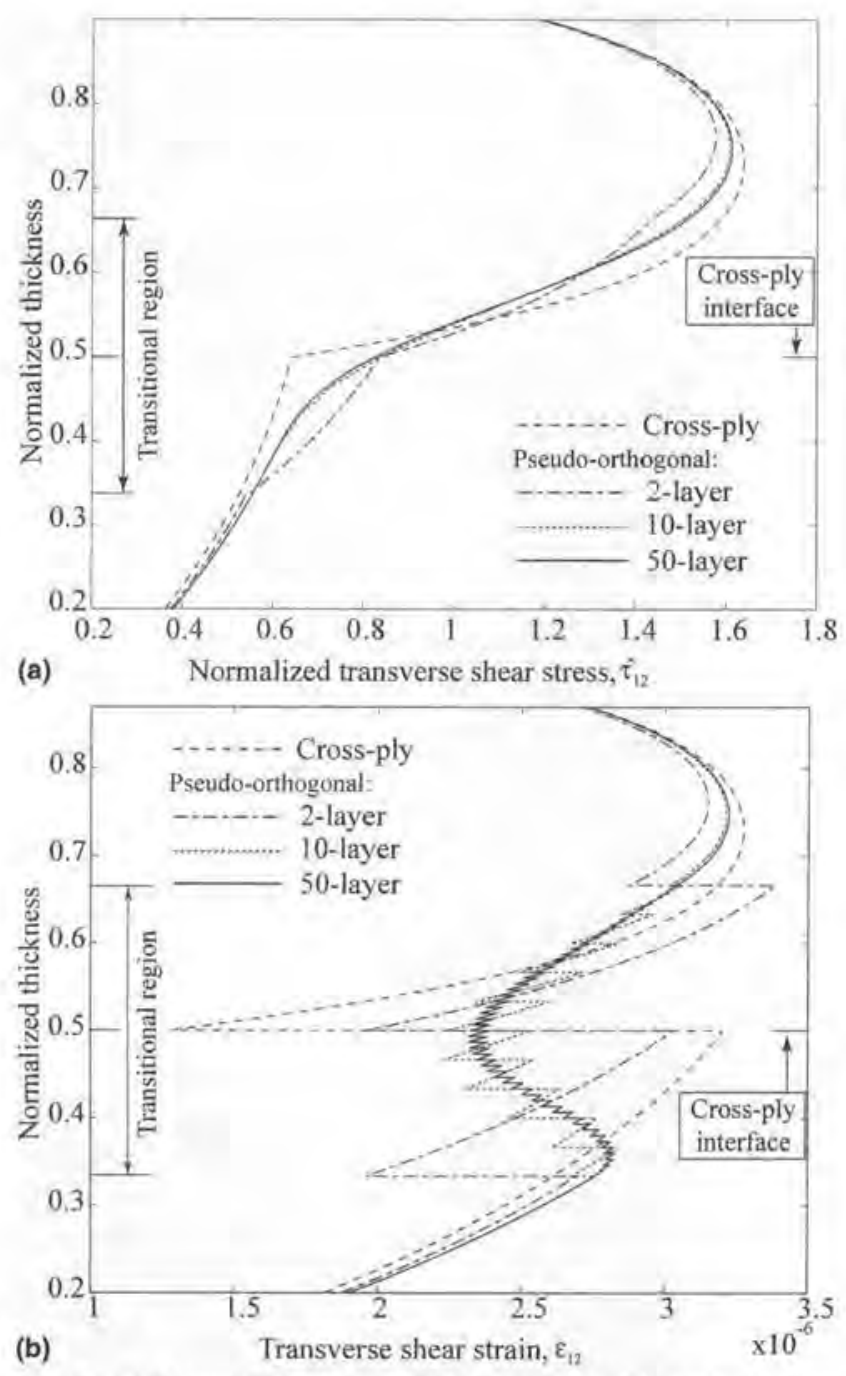

FIG. 7. (a) Normalized transverse shear stress and (b) strain distributions over the cross-section of the laminate with selected numbers of lamina in the transitional region.

Next, consider the transverse shear stress and strain distribution over the laminate cross-section. Unlike the normal stress and strain, the transverse shear stress remains continuous over the interfaces, whereas the transverse strain is discontinuous (Appendix B). As expected, this reaction is captured by the numerical simulations. The numerical results suggest that the transverse shear stress distribution is almost independent of the number of laminae in the transitional region [Fig. 7(a)]. However, the transverse shear strain shows a similar response to that of the normal stress, including a discontinuity over the interfaces. The magnitude of the discontinuity decreases with the increasing number of Iaminae in the transitional region [Fig. 7(b)]. A distinct redistribution of the shear strain is observed when introducing the pseudo-orthogonal structure compared with the cross-ply structure. The maximum transverse shear strain in the transitional region of the pseudo-orthogonal

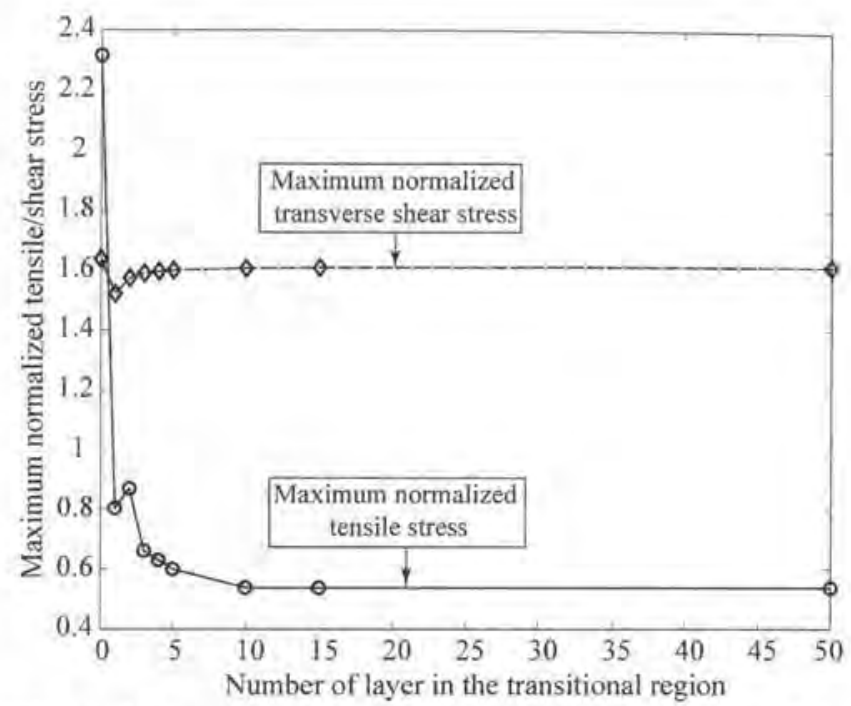

FIG. 8. Maximum normalized normal stress and transverse shear stress in the cross-section as a function of the number of laminae in the transitional region. The markers correspond to computed data points; lines are guide-for-the-eye only to demonstrate the trend.

structure is in general lower than that of the strain level of the cross-ply laminate [Fig. 7(b)]. In particular, by adopting the pseudo-orthogonal structure, the transverse shear strain in the structure is rearranged such that a part of the highest shear deformation concentrated in the $90^{\circ}$ layer near the interface is shifted to the transitional region. This shift releases a portion of the material from bearing excessive transverse shear deformation and achieves a more uniform transverse shear strain field.

So far, the thickness of the transitional region has been assumed to be constant. We will now investigate how the thickness of the transitional region influences the structural response. Three models with constant overall thickness, but various thickness of the transitional region $(33,20$, and $10 \%$ of the overall laminate thickness), will be investigated. The number of laminae in the transitional region is fixed to 10 laminae (estimation for the elytron sample). The results suggest that the maximum normal stress decreases with increasing thickness of the transitional region (Fig. 9). As expected, the magnitude of the jump remains constant [see Eq. (A11) in Appendix B]. The result suggests that even when the thickness of the transitional region is only $10 \%$ of the entire structure, significant advantages of the pseudoorthogonal structure can be obtained. This thickness of the transitional region closely corresponds to that of the biomaterial studied; in the endocuticle of the elytron, the thickness of the unidirectional layer is approximately $1 \mu \mathrm{m}$ and the transitional region is 0.1 to $0.2 \mu \mathrm{m}$ (5-10\% of the overall thickness of the pseudo-orthogonal structure). As shown in Fig. 8, with decreasing thickness of the transitional region, the pseudo-orthogonal structure's ability to reduce the magnitude of the discontinuity in 


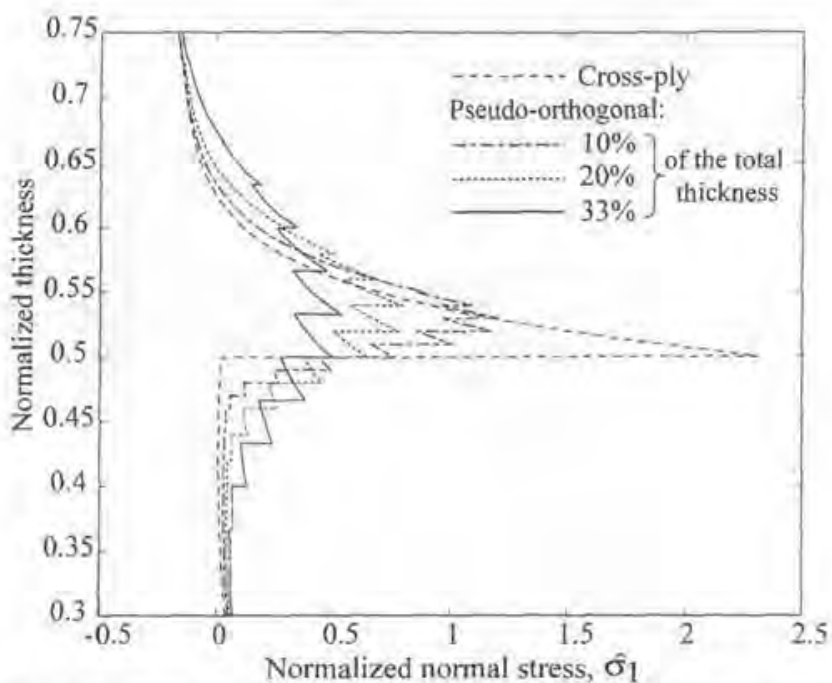

FIG. 9. Normalized normal stress distributions over the cross-section with selected transitional region thicknesses $(10,20$, and $33 \%$ transitional region thickness to that of the overall laminate) compared with a cross-ply Iaminate without transitional region.

the normal stress and shear strain is reduced. However. in practical engineering application, a thin transitional region may not be an optimum mechanical solution.

\section{Fracture mechanics considerations}

More insight to the structural response may be gained by considering the interfacial fracture resistance. The strain energy release rate, $G$, is typically used as a measure of the "crack driving force." The strain energy release rate is a structural parameter, determined by the applied load, the stiffness, and geometry of the structure, and the characteristics of the crack. Fracture occurs if $G \geq G_{c}$, where $G_{c}$ is the critical energy release rate or the fracture toughness. This is a material property, thus its value can only be determined via experimental measurements of the structure investigated. We will limit the investigation here to the structural property $G$, and we will show that $G$ decreases when the stiffness mismatch between two adjacent layers decreases. In other words, a layered structure is more resistant to fracture when the stiffness mismatch between two adjacent layers is low. A full investigation involving the measurement of the fracture resistance is beyond the scope of the current work.

For simplicity, we will limit the analysis to a split beam, split with a crack of length $a$ in the geometric centerline, subjected to tensile loads [An interface is always associated with mode-mixity. The load case considered here corresponds to a dominate mode II loading (in-plane shear). To keep the analysis simple, we do not decompose the results into mode I ("crack opening") and mode II.], as shown in Fig. 10. This simple model captures the basic features of the laminated biocomposite. Within the framework of LEFM, the strain energy release rate $(G)$ of the structure with the applied load

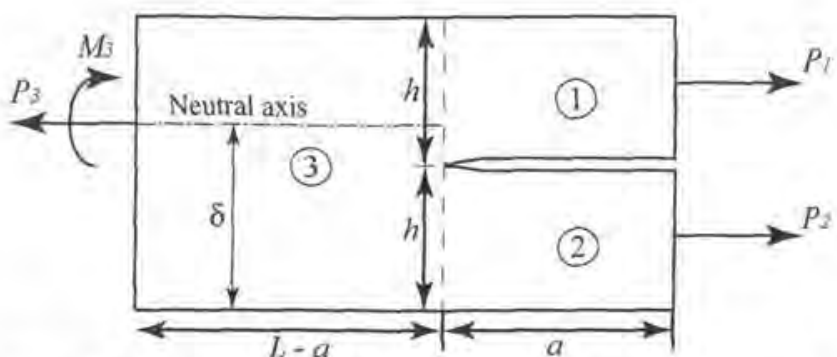

FIG. 10. Model of an equally split beam under horizontal loads. $P_{\text {i }}$ $(i=1,2)$ is the tensile force applied on segment $i$ of the beam; $h$ is the half thickness of the beam, $a$ is the initial crack length, and $L$ is the total length of the beam.

(as shown in Fig. 10) can be expressed as (detailed in Appendix C):

$$
G=\frac{1}{t}\left[\frac{P_{1}^{2}}{2 E_{1} A_{1}}+\frac{P_{2}^{2}}{2 E_{2} A_{2}}-\frac{P_{3}^{2}}{2 E_{3} A_{3}}-\frac{M_{3}^{2}}{2 D_{3}}\right],
$$

where $t$ is the thickness of the beam and subscript $i$ indicates the segment $i(i=1,2,3)$ of the beam. Furthermore, $E_{\mathrm{i}}$ is the elastic modulus and $A_{\mathrm{i}}$ is the area of the cross-section of each segment. $D_{3}$ is the flexural stiffness, defined in Appendix C, $P_{\mathrm{i}}$ is the in-plane force in each segment, and $M_{3}$ is the resultant bending moment, as defined by Fig. 10. The normalized strain energy release rate

$$
\hat{G}=G \frac{t^{2} h E_{3}}{P_{1}^{2}+P_{2}^{2}},
$$

as a function of the stiffness mismatch, $E_{1} / E_{2}$, between the adjacent materials is shown in Fig. 11 for a range of load combinations, $\beta=P_{1} / P_{2}$. It is clear from the figure that an increased stiffness mismatch results in an increased strain energy release rate for the considered load condition (Fig. 11). Similar results are obtained for alternative load conditions (e.g., shear-force, bending moments, and combined loading), but are omitted here for brevity.

These results can be used to compare the cross-ply and pseudo-orthogonal structures. The cross-ply structure is characterized by a large stiffness mismatch over the interface compared with the pseudo-orthogonal structure. Thus, for a given load, the cross-ply will result in a larger strain energy release rate than that of the pseudo-orthogonal structure. Furthermore, with the increasing number of transitional lamina, the strain energy release rate will be further reduced as a direct result of the reduction of the stiffness mismatch across the interface (Fig. 11). Moreover, the results can be extended towards the helicoidal structure in the exo- and mesocuticles. In the helicoidal regions, the gradual rotation of the lamina imposes a consistent small stiffness change between the lamina. According to the model presented here, this effect will result in a relatively high fracture toughness resistance throughout each region. 


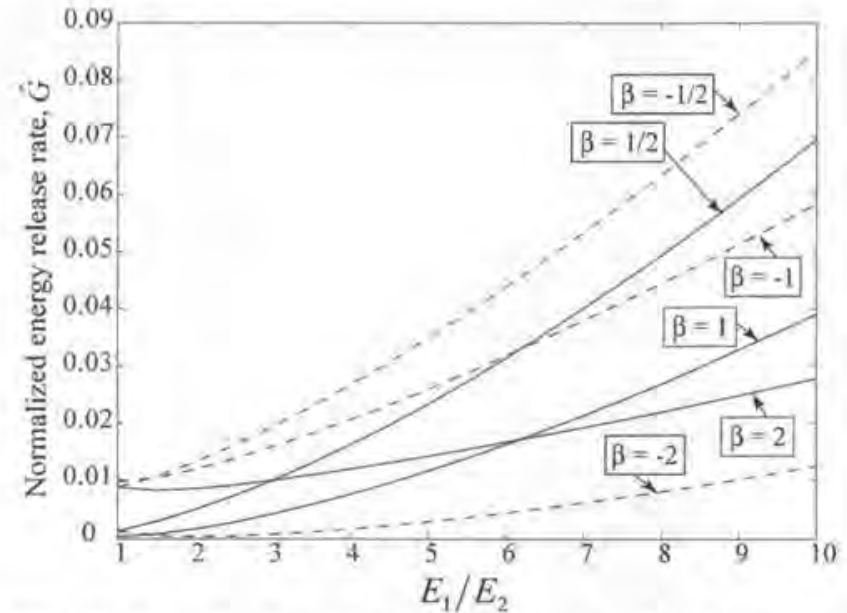

FIG. 11. Normalized strain energy release rate of the beam as a function of stiffness mismatch across the interface $\left(E_{1} / E_{2}\right)$, and selected ratios of applied load to segment 1 and $2\left(\beta=P_{1} / P_{2}\right)$.

\section{Synopsis}

The modeling results suggest that the pseudo-orthogonal structure improves the mechanical behavior of the entire structure. The maximum normal stress and the jump in both the normal stress and transverse strain are reduced when the transitional region is introduced. The simple fracture mechanics analysis indicated that the interfacial strain energy release rate is reduced when the difference in stiffness between two adjacent layers is reduced. Assuming that the intrinsic strength of the interface remains unchanged (since the interfacial strength depends mostly on the "matrix material" that keeps the layers together), a decreasing energy release rate corresponds to an increase in structural strength. This result is in agreement with analysis of composite structures that suggest that a lower stiffness mismatch between adjacent layers increases the debonding strength of the structure. $^{36}$ Thus, we believe that the pseudo-orthogonal structure is a more durable structure than the traditional cross-ply structure. However, information about the allowable load (e.g., force, stress, or deformation) along specified directions or the interfacial strength is not available from the literature (to the knowledge of the authors). Systematic experiments and rigorous micromechanical analysis must be carried out to obtain such information, including the allowable normal stress or strain in principle direction and shear stress or strain inplan and out-of-plan. This is beyond the scope of current study and will be investigated in a future study.

The coexistence of the pseudo-orthogonal structure and helicoidal structure in the exoskeleton suggests a competition between the in-plane isotropy and the interfacial strength in nature's design and manufacturing of the biocomposite.

(i) The pseudo-orthogonal structure exhibits an overall response similar to that of a cross-ply structure, e.g., in-plane isotropy. The relatively low resistance of the traditional cross-ply structure to interfacial fractures is mitigated by the transitional region.

(ii) The helicoidal structure exhibits (almost) inplane isotropy, ${ }^{3}$ but there is a coupling between stretching and bending that may induce large deflection and stresses. However, the helicoidal structure has a relatively high resistance towards interfacial debonding, because the stiffness difference between each layer is low.

Thus, we propose that the helicoidal structure is designed as a compromise between the need for high interfacial strength of the laminate and isotropic response. The presence of the two structures in the exoskeletons is most likely a manifestation of the competition between the two performance aspects, where the pseudo-orthogonal structure provides an overall isotropic structure with "acceptable" interfacial strength, whereas the helicoidal structure provides overall high interfacial fracture strength with an acceptable isotropic response. [An additional consideration is that the pseudoorthogonal region (i.e., the endocuticle) constitutes at least half of the total exoskeleton thickness (Table I). This effect contributes to an overall isotropic response of the exoskeleton.]

Finally, the three locations of the $P$. japonica body (pronotum, leg, and elytron) that were examined in this study display similar exoskeletal morphology, possibly due to the similarity of their mechanical-loading environments. These three areas are constantly exposed to the external environment, and their main function is believed to protect the beetle against external loads during movement, attack, and defense, in addition to the leg's load-bearing function. The exoskeletons are likely subjected to bending, shearing, and compression as investigated above. Other body parts may experience different loading conditions and thus have different morphology in the exoskeleton. For example, the chitin fiber orientation was found to be approximately perpendicular to the external surface in the head articulation cuticles, possibly due to the unique mechanical environment (e.g., friction and wear). ${ }^{22}$ All of these observations support the notion that the exoskeletal structure adapts to the local mechanical and chemical environments. A similar strategy is expected for the mechanical properties of other exoskeletons, as for example shown in a recent study in armored fish scale. $^{13}$

\section{CONCLUSIONS}

This study investigated the exoskeletal microstructure of a common insect, $P$. japonica (Japanese beetle), and the implications the observed microstructure has on the exoskeleton's mechanical performances. 
Image analysis via SEM and TEM revealed a common morphology in cuticles from the pronotum, leg, and elytron. All exoskeletons consist of four regions, including (from external surface and inwards and corresponding to increasing thickness) epicuticle, exocuticle, mesocuticle, and endocuticle. The latter three regions are the load-bearing structures and are comprised of chitin-protein fiber layers orientated parallel to the cuticle surface. The chitin fibers in the exocuticle and mesocuticle are organized in a helicoidal structure, which is characterized by layers stacked with a small rotational angle relative to their adjacent layers. The endocuticle has a distinct pseudo-orthogonal pattern, which is characterized by a thin transitional helicoidal region inserted between two orthogonally stacked layers.

Idealized models of orthogonally layered structures with and without the helicoidal transitional region (corresponding to the pseudo-orthogonal and conventional cross-ply laminates respectively) were developed for evaluating the endocuticle's mechanical response within the linear elastic range. The mechanical response of the conventional cross-ply laminate includes discontinuity of the normal stress and transverse shear strain at the interfaces of the orthogonal laminae due to the discontinuous material properties (assuming laminate theory within the framework of continuum mechanics). The introduction of a pseudo-orthogonal structure results in a redistribution of the stress and strain fields, including smaller discontinuities between the layers and more uniform stress and strain distribution over the cross-section. The pseudo-orthogonal structure results in reduced maximum tensile stress and transverse shear stress in the across-section. The magnitude of the discontinuity (jump) in the normal stress and shear strain is significantly reduced as well. Furthermore, the interfacial strain energy release rate of the laminate is lower in the pseudo-orthogonal structure compared to the cross-ply laminate, suggesting that the pseudo-orthogonal structure may be more resistant to fracture.

The coexistence of the pseudo-orthogonal and helicoidal structure in the exoskeleton suggests a competition between the in-plane isotropy and the interfacial strength in nature's design and manufacturing of the biocomposite. We believe that the pseudo-orthogonal structure provides an overall isotropic structure with an acceptable interfacial strength, whereas the helicoidal structure provides overall high interfacial fracture strength with an acceptable isotropic response.

In conclusion, these results reveal interesting aspects of the strategy of the nature in designing and manufacturing functional biomaterial systems. These observations may be used to inspire and improve man-made materials and structures.

\section{ACKNOWLEDGMENTS}

We would like to thank Dr. Kirk Czymmek and Ms. Shannon Modla at the Delaware Biotechnology Institute for helping in the sample preparations and SEM/TEM observations. We gratefully acknowledge support from the United States Army Research Office and the National Institutes of Health (Grants AR054385 and P20RR016458).

\section{REFERENCES}

1. S.O. Andersen: Biochemistry of insect cuticle: Annu. Rev. Entomol. 24, 29 (1979).

2. A.C. Neville: Biology of the Arthropod Cuticle, 2nd ed. (SpringerVerlag, New York, 1975),

3. L. Cheng, L.Y. Wang, and A.M. Karlsson: Image analyses of two crustacean exoskeletons and implications of the exoskeletal microstructure on the mechanical behavior. J. Mater. Res. 23(11). 2854 (2008).

4. T.D. Schultz and M.A. Rankin: The ultrastructure of the epicuticular interference reflectors of tiger beetles (Cicindela). J. Exp Biol. 117(Jul), 87 (1985),

5. J.F.V. Vincent and U.G.K. Wegst: Design and mechanical properties of insect cuticle. Arthropod Struct. Dev. 33(3), 187 (2004).

6. U.G.K. Wegst and M.F. Ashby: The mechanical efficiency of natural materials. Philos. Mag. 84(21), 2167 (2004).

7. T. Nishino, R. Matsui, and K. Nakamae: Elastic modulus of the crystalline regions of chitin and chitosan. J.Polym. Sci., Part B: Polym. Phys. 37(11), 1191 (1999).

8. J.F.V. Vincent: Arthropod cuticle: A natural composite shell system. Composites Part A 33(10), 1311 (2002).

9. W. Xu, P.J. Mulhern, B.L. Blackford, M.H. Jericho, and I. Templeton: A new atomic-force microscopy technique for the measurement of the elastic properties of biological-materials. Scanning Microsc. 8(3), 499 (1994).

10. A.C. Neville, D.A.D. Parry, and J. Woodheadgalloway: Chitin crystallite in arthropod cuticle. I. Cell Sci. 21(1), 73 (I976).

11. J.F.V. Vincent: Insect cuticle: A paradigm for natural composites. Symp. Soc. Exp. Biol. 34, 183 (1980).

12. Y. Bouligan: Twisted fibrous arrangements in biological-materials and cholesteric mesophases. Tissue Cell 4(2), 189 (1972).

13. B.J.F Bruet, J.H. Song, M.C Boyce, and C. Ortiz: Materials design principles of ancient fish armour. Nat. Mater. 7(9), 748 (2008).

14. S. Gunderson and R, Schiavone: The insect exoskeleton-A natural structural composite, JOM 41(11), 60 (1989).

15. H.R. Hepburn and A. Ball: Structure and mechanical properties of beetle shells. J. Mater. Sci. 8(5), 618 (1973).

16. D. Raabe, C. Sachs, and P. Romano: The crustacean exoskeleton as an example of a structurally and mechanically graded biological nanocomposite material. Acta Mater. 53(15), 4281 (2005).

17. B. Zelazny and A.C. Neville: Quantitative studies on fibril orientation in beetle endocuticle. $I$. Insect Physiol. 18(11), 2095 (1972).

18. A. Sugawara, T. Nishimura, Y. Yamamoto, H. Inoue, H. Nagasawa, and T. Kato: Self-organization of oriented calcium carbonate/polymer composites: Effects of a matrix peptide isolated from the exoskeleton of a crayfish. Angew. Chem. Int Ed. 45(18), 2876 (2006).

19. S. Weiner and L. Addadi: Design strategies in mineralized biological materials. J. Mater. Chem. 7(5). 689 (1997),

20. B.J.F. Bruet, H.J. Qi, M.C. Boyce, R. Panas, K. Tai, L. Frick, and C. Ortiz: Nanoscale morphology and indentation of individual 
nacre tablets from the gastropod molluse Trochus niloticus, I. Mater. Res. 20(9), 2400 (2005).

21. A.C. Neville and B.M. Luke: Molecular architecture of adult locust cuticle at the electron microscope level. Tissue Cell 1(2), 355 (1969).

22. N. Barbakadze, S. Enders, S. Gorb, and E. Arze Local mechanical properties of the head articulation cuticle in the beetle Pachnoda marginata (Coleoptera, Scarabaeidae). I. Exp. Biol. 209(4), 722 (2006).

23. P.Y, Chen, A.Y.M. Lin, J. McKittrick, and M.A. Meyers: Structure and mechanical properties of crab exoskeletons. Acta Biomater. $4(3), 587$ (2008).

24. V.A. Lindley: A new procedure for handling impervious biological specimens. Microsc. Res. Tech. 21(4), 355 (1992).

25. E.S, Reynolds: Use of lead citrate at high $\mathrm{ph}$ as an electronopaque stain in electron microscopy. J. Cell Biol. 17(1), 208 (1963).

26. S.N. Gorb: Porous channels in the cuticle of the head-arrester system in dragon/damselflies (Insecta: Odonata). Microsc. Res. Tech. 37(5-6), 583 (1997).

27. S. Hild, O. Marti, and A. Ziegler: Spatial distribution of calcite and amorphous calcium carbonate in the cuticle of the terrestrial crustaceans Porcellio scaber and Armadillidium vulgare. $J$. Struct. Biol. 163(1), 100 (2008).

28. F. Bobelmann, P. Romano, H. Fabritius, D. Raabe, and M. Epple: The composition of the exoskeleton of two crustacea: The American lobster Homarus americanus and the edible crab Cancer pagurus. Thermochim. Acta 463(1-2), 65 (2007).

29. P. Fratzl, I. Burgert, and H.S. Gupta: On the role of interface polymers for the mechanics of natural polymeric composites. Phys. Chem. Chem. Phys. 6(24), 5575 (2004).

30. J.E. Hillerton: Electron-microscopy of fibril-matrix interactions in a natural composite, insect cuticle. J.Mater. Sci. 15(12), 3109 (1980).

31. H.O. Fabritius, C. Sachs, P.R. Triguero, and D. Raabe: Influence of structural principles on the mechanics of a biological fiberbased composite material with hierarchical organization: The exoskeleton of the lobster Homarus americanus. Adv. Mater, 21(4), 391 (2009).

32. A. Fritsch, L. Dormieux, C. Hellmich, and J. Sanahuja: Micromechanics of crystal interfaces in polycrystalline solid phases of porous media: Fundamentals and application to strength of hydroxyapatite biomaterials. J. Mater. Sci. 42(21), 8824 (2007).

33. C. Hellmich, J.F. Barthelemy, and L. Dormieux: Mineral-collagen interactions in elasticity of bone ultrastructure-A continuum micromechanics approach. Eur. I. Mech. A, Solids 23(5), 783 (2004).

34. J.N. Reddy: Mechanics of Laminated Composite Plates: Theory and Analysis, 2nd ed. (CRC Press, New York, 1997).

35. ABAQUS 6.7 (ABAQUS Inc., Pawtucket, RI, 2007).

36. W.J. Renton and J.R. Vinson: On the behavior of bonded joints in composite material structures. Eng. Fract. Mech. 7, 41 (1975).

37. J.W. Hutchinson and Z. Suo: Mixed-mode cracking in layered materials. Adv. Appl. Mech. 29, 63 (1992).

\section{APPENDIX A: CLASSIC LAMINATE THEORY}

Here, we present a short summary of the classic laminate theory. This theory can now be found in many text books, and in what follows we use notations consistent with that of Reddy. ${ }^{34}$

The linear-elastic stiffness of an individual lamina can be expressed via the stiffness matrix, $\mathbf{Q}{ }^{34}$

$$
\mathbf{Q}=\left[\begin{array}{cccccc}
Q_{11} & Q_{12} & Q_{13} & 0 & 0 & 0 \\
Q_{12} & Q_{22} & Q_{23} & 0 & 0 & 0 \\
Q_{13} & Q_{23} & Q_{33} & 0 & 0 & 0 \\
0 & 0 & 0 & Q_{44} & 0 & 0 \\
0 & 0 & 0 & 0 & Q_{55} & 0 \\
0 & 0 & 0 & 0 & 0 & Q_{66}
\end{array}\right]
$$

with the components $Q_{\mathrm{ij}}$ :

$$
\begin{gathered}
Q_{11}=E_{1}\left(1-v_{23} v_{32}\right) / \Delta, Q_{22}=E_{2}\left(1-v_{31} v_{13}\right) / \Delta, \\
Q_{33}=E_{3}\left(I-v_{12} v_{21}\right) / \Delta, Q_{44}=G_{23}, Q_{55}=G_{13}, Q_{66}=G_{12},
\end{gathered}
$$

$$
\begin{aligned}
Q_{12} & =E_{1}\left(v_{21}+v_{31} v_{23}\right) / \Delta, Q_{13}=E_{1}\left(v_{31}+v_{21} v_{32}\right) / \Delta, \\
Q_{23} & =E_{2}\left(v_{32}+v_{12} v_{31}\right) / \Delta, \\
\Delta & =1-v_{12} v_{21}-v_{23} v_{32}-v_{31} v_{13}-2 v_{21} v_{32} v_{13},
\end{aligned}
$$

where $E_{1}, E_{2}$, and $E_{3}$ are Young's moduli along the lamina's principle axis; $G_{12}, G_{13}$, and $G_{23}$ are the shear moduli; and $v_{i j}(i, j=1,2,3)$ are Poisson's ratios. ${ }^{34}$

When the laminae's principle axes are not aligned with the global coordinate system in which the structure is described, then a coordinate transformation is necessary to express the elastic properties of laminae with various orientations within the global coordinate system. The transformed stiffness matrix $\overline{\mathbf{Q}}$ can be expressed in matrix form as

$$
\overline{\mathbf{Q}}=\mathbf{T}^{-1} \mathbf{Q T},
$$

where $\mathbf{T}$ is the transformation matrix

$$
\mathbf{T}=\left[\begin{array}{cccccc}
m^{2} & n^{2} & 0 & 0 & 0 & 2 m n \\
n^{2} & m^{2} & 0 & 0 & 0 & -2 m n \\
0 & 0 & 1 & 0 & 0 & 0 \\
0 & 0 & 0 & m & -n & 0 \\
0 & 0 & 0 & n & m & 0 \\
-m n & m n & 0 & 0 & 0 & m^{2}-n^{2}
\end{array}\right]
$$

with $m=\cos \theta, n=\sin \theta$, where $\theta$ denotes the lamina orientation with respect to the global coordinate system. ${ }^{34}$

\section{APPENDIX B: DEFORMATION CONTINUITY AND STRESS EQUILIBRIUM IN LAMINATED COMPOSITES}

Here, we will give a qualitative explanation to the discontinuities observed in the normal stress and shear strain over the interfaces as presented in Sec. III. B, by using a simple analytical approach.

The integrity of the laminated composite structures requires the continuity of the displacement field. This process can be expressed as (Fig. Al)

$$
u_{1}^{k}\left(h_{k}^{-}\right)=u_{1}^{k+1}\left(h_{k}^{+}\right), u_{2}^{k}\left(h_{k}^{-}\right)=u_{2}^{k+1}\left(h_{k}^{+}\right),
$$


where $u_{i}^{k}\left(u_{i}^{k+1}\right)$ is the displacement of material of $k^{\text {th }}\left(k^{\text {th }+1}\right)$ lamina in direction $i=1,2, h_{\mathrm{k}}$ is the distance from the laminate mid-plane to the interface between the laminae $k$ and $k+1$, and $h_{k}^{+}, h_{k}^{-}$denote the positions infinitely close to the interface in the two neighboring laminae. From Eq. (A5), it follows that the normal strain fields across the lamina interface are continuous, since it holds that:

$$
\varepsilon_{11}^{k}\left(h_{k}^{-}\right)=\frac{\partial u_{1}^{k}}{\partial x_{1}}\left(h_{k}^{-}\right)=\frac{\partial u_{1}^{k+1}}{\partial x_{1}}\left(h_{k}^{+}\right)=\varepsilon_{11}^{k+1}\left(h_{k}^{+}\right) .
$$

Moreover, Newton's 3rd law requires that the forces in the exposed interfaces are equal in magnitude but opposite in direction, when making an imaginary cross-sectioning of the interface. That is, $F^{k}\left(h_{k}^{-}\right)+F^{k+1}\left(h_{k}^{+}\right)=0$, where $F$ corresponds to either the shear or normal force of the cross-section. Assuming that $A$ is the area that the force is acting upon and noting that $A \neq 0$, this result implies (using the sign convention adopted in Fig. A1):

$$
\tau_{12}^{k}\left(h_{k}^{-}\right)=\tau_{12}^{k+1}\left(h_{k}^{+}\right), \sigma_{22}^{k}\left(h_{k}^{-}\right)=\sigma_{22}^{k+1}\left(h_{k}^{+}\right),
$$

where $\tau_{12}^{j}$ and $\sigma_{22}^{j}$ are the transverse shear stress and normal stress, respectively, at the $j^{\text {th }}$ lamina.

The constitutive equations relate the strains to the stresses:

$$
\begin{gathered}
\sigma_{11}=Q_{11} \varepsilon_{11}+Q_{12} \varepsilon_{22}, \sigma_{22}=Q_{12} \varepsilon_{11}+Q_{22} \varepsilon_{22}, \\
\varepsilon_{12}=\frac{1}{2 G_{12}} \tau_{12},
\end{gathered}
$$

where $Q_{i j}$ are the stiffnesses presented in Appendix A. For simplicity, the superscript indicating the lamina, $k$ or $k+1$, is omitted in Eqs. (A8) and (A9). Because the item $Q_{12} \varepsilon_{22}$ is much smaller than $Q_{12} \varepsilon_{22}$, Eq. (A8) can be simplified to:

$$
\sigma_{11} \cong Q_{11} \varepsilon_{11}
$$

Substituting Eq. (A9) into Eq. (A5) results in

$$
\frac{\sigma_{11}^{k}\left(h_{k}^{-}\right)}{\sigma_{11}^{k+1}\left(h_{k}^{+}\right)}=\frac{Q_{11}^{k}}{Q_{11}^{k+1}} \text {. }
$$

Laminae with different orientations [orientation is $\theta^{k}$ for the $k^{\text {th }}$ lamina and $\theta^{k+1}$ for the $(k+1)^{\text {th }}$ lamina] have

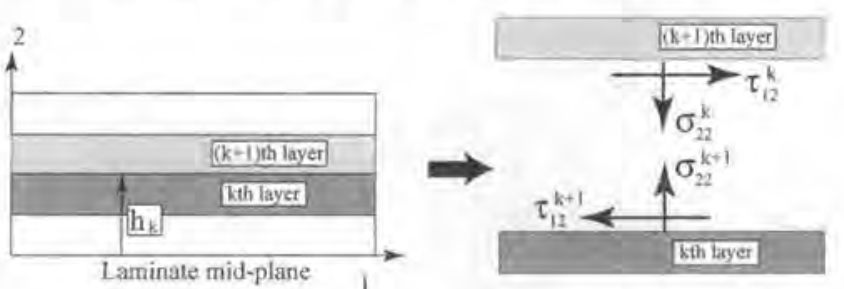

FIG. Al. Illustration for establishing the interfacial stresses in the laminate. $h^{k}$ is the distance from the mid-plane to the interface between the $k^{\text {th }}$ and the $(k+1)^{\text {th }}$ laminae. different elastic properties in the global coordinate system, as shown in Appendix A. Thus, over the interface of two laminae with different orientations, $Q_{11}^{k} / Q_{11}^{k+1} \neq 1$, and it follows from Eq. (A10) that a discontinuity in the normal stress $\sigma_{11}$ ensues.

Likewise, a discontinuity in the transverse shear strain over the interface is obtained based on Eqs. (A6) and (A9):

$$
\frac{\varepsilon_{12}^{k}\left(h_{k}^{-}\right)}{\varepsilon_{12}^{k+1}\left(h_{k}^{+}\right)}=\frac{G_{12}^{k+1}}{G_{12}^{k}} \neq 1 .
$$

\section{APPENDIX C: LINEAR ELASTIC FRACTURE MECHANICS}

The derivation of the strain energy release rate, $G$, of an interfacial crack subjected to tensile forces as shown in Fig. 10 will be outlined here. The interface considered is between two dissimilar materials with elastic modulus $E_{1}$ and $E_{2}$ (segment 1 and 2, respectively), as shown in Fig. 10. The intact segment (segment 3 ) is assumed to be a perfectly bonded layered composite of the two materials. All of the definitions and derivations are based on the LEFM consistent with the presentation by Hutchinson and Suo. ${ }^{37}$ The strain energy release rate can be defined as follows:

$$
G=\frac{1}{t} \frac{d U}{d a}=\frac{1 d \sum U_{i}}{t}, i=1,2,3,
$$

where $t$ is the thickness (out of plane) and $U_{\mathrm{i}}$ refers to the strain energy (i.e., the potential energy stored in a body by elastic deformation) stored in the three segments in the structure (Fig. 10). For segments 1 and 2, the strain energy is derived based on linear elastic assumption $^{37}$ :

$$
U_{i}=\frac{P_{i}^{2} a}{2 E_{i} A_{i}}, i=1,2,
$$

where $P_{\mathrm{i}}$ is the force applied to segment $i$, and $E_{\mathrm{i}}$ and $A_{i}$ correspond to the elastic modulus and cross-sectional area of segment $i(i=1,2)$. For segment 3, the strain energy is as follows:

$$
U_{3}=\frac{P_{3}^{2}(L-a)}{2 E_{3} A_{3}}+\frac{M_{3}^{2}(L-a)}{2 D_{3}},
$$

where $P_{3}$ and $M_{3}$ are the force and bending moment applied on segment $3, E_{3}, A_{3}$, and $D_{3}$ correspond to the elastic modulus, the cross-section area, and the flexural stiffness of segment 3 , respectively. It holds that:

$$
\begin{gathered}
P_{3}=P_{1}+P_{2} \\
M_{3}=P_{1}\left(\frac{3}{2} h-\delta\right)-P_{2}\left(\delta-\frac{1}{2} h\right) .
\end{gathered}
$$




$$
\begin{gathered}
E_{3} A_{3}=E_{1} A_{1}+E_{2} A_{2} \\
D_{3}=E_{1}\left[\frac{1}{12} t h^{3}+t h\left(\frac{3}{2} h-\delta\right)^{2}\right] \\
+E_{2}\left[\frac{1}{12} t h^{3}+t h\left(\delta-\frac{1}{2} h\right)^{2}\right],
\end{gathered}
$$

where $\delta$ is the distance of the neutral axis of segment 3 from the bottom of the segment (Fig. 10):

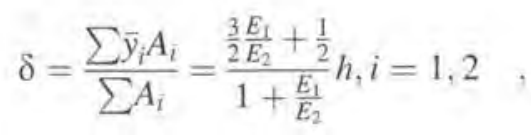

where $\bar{y}_{i}$ represents the distance from the bottom of segment 3 of the centroid of segment $i(i=1,2)$, which constitute segment 3 .

Finally, the strain energy release rate can be written as follows:

$$
G=\frac{1}{t}\left[\frac{P_{1}^{2}}{2 E_{1} A_{1}}+\frac{P_{2}^{2}}{2 E_{2} A_{2}}-\frac{P_{3}^{2}}{2 E_{3} A_{3}}-\frac{M_{3}^{2}}{2 D_{3}}\right] .
$$

